Published in final edited form as:

Brain Res. 2008 January 23; 1190: 15-22.

\title{
Specific AAV Serotypes Stably Transduce Primary Hippocampal and Cortical Cultures with High Efficiency and Low Toxicity
}

\author{
Nicolas C. Royo ${ }^{1}$, Luk H. Vandenberghe ${ }^{2,5}$, Jing-Yuan Ma ${ }^{1}$, Alisse Hauspurg ${ }^{1}$, LiYa Yu ${ }^{1}$, \\ Margaret Maronski ${ }^{3,4}$, Julie Johnston ${ }^{2}$, Marc A. Dichter ${ }^{3,4}$, James M. Wilson ${ }^{2}$, and Deborah \\ J. Watson ${ }^{1, \#}$ \\ 1 Department of Neurosurgery, University of Pennsylvania School of Medicine, Philadelphia PA 19104 \\ 2 Gene Therapy Program, Department of Pathology and Laboratory Medicine, University of Pennsylvania \\ School of Medicine, Philadelphia PA 19104
}

3 David Mahoney Institute for Neurological Sciences, University of Pennsylvania School of Medicine, Philadelphia PA 19104

4 Department of Neurology, University of Pennsylvania School of Medicine, Philadelphia PA 19104

5 Molecular Medicine, Katholieke Universiteit Leuven, Kapucijnenvoer 33, B-3000 Leuven, Belgium

\begin{abstract}
Most current methods of gene delivery for primary cultured hippocampal neurons are limited by toxicity, transient expression, the use of immature neurons, and/or low efficiency. We performed a direct comparison of seven serotypes of adeno-associated virus (AAV) vectors for genetic manipulation of primary cultured neurons in vitro. Serotypes 1,2, 7,8 and 9 mediated highly efficient, nontoxic, stable long-term gene expression in cultured cortical and hippocampal neurons aged 0-4 weeks in vitro; serotypes 5 and 6 were associated with toxicity at high doses. AAV1 transduced over $90 \%$ of all cells with approximately $80 \%$ of the transduced cells being neurons. The method was readily adapted to a high-throughput format to demonstrate neurotrophin-mediated neuroprotection from glutamate toxicity in cultured neurons at 2 weeks in vitro. These vectors should prove highly useful for efficient overexpression or downregulation of genes in primary neuronal cultures at any developmental stage.
\end{abstract}

\author{
Keywords \\ viral vector; gene delivery; cultured neurons
}

\section{Introduction}

Cultures of dissociated primary neurons and astrocytes are frequently used by neurobiologists to study neuronal physiology and pathophysiology. A variety of methods has been used for gene delivery into cultured neurons, including recombinant Sindbis, SV40 or Semliki-forest viral vectors as well as plasmid transfection using calcium phosphate, commercially available

\footnotetext{
\#Address correspondence to: Deborah J. Watson, PhD., 371A Stemmler Hall/6071, Department of Neurosurgery, University of Pennsylvania School of Medicine, Philadelphia, PA 19104, Phone: 215-662-7931, Fax: 215-349-8157, Email: djw3@mail.med.upenn.edu.

Publisher's Disclaimer: This is a PDF file of an unedited manuscript that has been accepted for publication. As a service to our customers we are providing this early version of the manuscript. The manuscript will undergo copyediting, typesetting, and review of the resulting proof before it is published in its final citable form. Please note that during the production process errors may be discovered which could affect the content, and all legal disclaimers that apply to the journal pertain.
} 
lipids, or electroporation. However, each of these methods is limited by one or more of the following drawbacks: very low efficiency, short-term gene expression, toxicity, inability to express common reporter genes, and/or the requirement to transfect immature neurons (reviewed in [7]). Although transient, low-efficiency gene expression is sufficient for some types of experiments, biochemical experiments require much higher efficiency.

Adeno-associated viral (AAV) vectors readily transduce neurons in vivo with low toxicity. Here, we report in vitro transduction and toxicity patterns for AAV vectors with 7 different serotypes and 4 AAV vectors with engineered capsids. Several of these vectors mediated efficient, stable, and nontoxic transduction of hippocampal and cortical neurons in vitro.

\section{Results}

\section{Tropism and efficiency}

Recombinant AAV vectors were generated using a CMV-GFP expression cassette as the genome and packaged using capsid sequences from AAV1, 2, 5, 6, 7, 8, or 9. To compare transduction efficiencies of the different AAV serotypes, a single dose of $2.0-2.5 \times 10^{11}$ genome copies (GC) of each vector was added to cultured rat hippocampal cells on day in vitro (DIV) 7. By DIV21, the cultures exposed to serotypes 1, 2, 7, 8 and 9 showed remarkable levels of transduction, approximately $80-94 \%$ of the cells in each culture (Table 1). Expression of GFP was detectable in both neurons and astrocytes by 1 week post-transduction and continued for the life of the culture (Fig. 1A-C).

Transduced neurons and astrocytes were identified by colocalizing intrinsic GFP fluorescence with anti-MAP2 immunostaining to label neurons or anti-GFAP immunostaining to label astrocytes. None of the vectors transduced neurons or astrocytes exclusively (Fig. 2; Table 1). AAV1 and AAV6 were the most neurotropic. Transduction by AAV1 resulted in $94.1 \%$ overall transduction; $\sim 80 \%$ of the transduced cells were neurons. For AAV6, overall transduction was decreased to $72 \%$ because lower doses were necessary due to toxicity (see below), but of the cells that were transduced $\sim 85 \%$ were neurons. AAV2, AAV7 and AAV8 transduced $54 \%$, $74 \%$ and $63 \%$ neurons, respectively ( $p>0.05$ vs. AAV1). In contrast, AAV9 transduced more astrocytes than neurons ( $43.2 \%$ neurons; $\mathrm{p}<0.005$ vs. AAV1). Transduction patterns were similar on cocultured cortical neurons and astrocytes (Fig. 1D-G).

The importance of the heparin-binding region for AAV2 transduction was tested by using an engineered variant (AAV2/hu29R) with SGNT replacing RGNR at the consensus RXXR heparin-binding motif. The heparin-binding region was found to be essential for neuronal and glial transduction by AAV2. Although no cells were transduced, hu29R was not toxic as determined by normal MAP2 and GFAP staining.

\section{Toxicity}

At a high dose of $2.5 \times 10^{11} \mathrm{GC}$, most cultures exposed to serotypes 1, 2, 7, 8 or 9 showed no neuronal toxicity as demonstrated by MAP2 immunoreactivity identical to untreated cultures. However, AAV5 and AAV6 were toxic to both neurons and astrocytes at a dose of $2.5 \times 10^{11}$ GC (Table 1). The toxicity was probably unrelated to the volume delivered (16-26 $\mu$ l) because the vehicle itself (PBS+5\% glycerol) was nontoxic up to quantities of $50 \mu \mathrm{l} /$ culture (data not shown). Lower doses $\left(2.5 \times 10^{9} \mathrm{GC}\right)$ of either AAV5 or AAV6 encoding CMV-GFP yielded $48-72 \%$ overall transduction and minimal toxicity to neurons at 2 weeks post-transduction. To determine the possible contribution of GFP toxicity, parallel experiments were performed with AAV5 and AAV6 encoding LacZ. Both AAV5-CMV-LacZ and AAV6-CMV-LacZ were toxic at 2 weeks post-transduction, suggesting that the capsid sequence rather than GFP overexpression is involved in AAV5 and AAV6 toxicity in vitro. 
To explore the role of the capsid sequence in toxicity, we altered specific amino acids in the AAV6 capsid. AAV1, which is nontoxic in these cultures, differs from AAV6 by 6 amino acids in the cap sequence, including the K531 residue critical for heparin binding [33]. Therefore, we tested whether AAV6 toxicity could be ameliorated by replacing individual amino acids in the AAV6 capsid with the corresponding AAV1 residue. Table 1 shows that the toxicity was blocked by mutating K531 to glutamine (K531E, AAV6.1), but not by a change at residue 129 that does not alter heparin binding (F129L, AAV6.2). Changing both residues simultaneously (AAV6.1.2) also blocked toxicity. Of note, alteration of the heparin-binding residues in AAV6 did not block transduction as it did with AAV2. This is consistent with a previous report showing that AAV2 but not AAV6 transduction is blocked by the addition of heparin, and that AAV2 and AAV6 do not compete for the same cellular receptors [13].

\section{Time course of expression}

GFP-expressing cells were clearly visible at $3 \mathrm{~d}$ post-transduction. Immunolabeling experiments revealed that the GFP-expressing cells were GFAP-positive astrocytes, regardless of the serotype used for transduction (AAV1, 2, 7, 8, 9). At 1-2 weeks post-transduction, both neurons and astrocytes showed robust GFP fluorescence after transduction by AAV1, 2, 7, 8 or 9 , indicating that the early expression in astrocytes and later expression in neurons is principally a result of hCMV promoter usage in each cell type (Fig. 2). Transduction levels did not change between 1 week post-transduction and later time points. Once present, GFP expression continued in both neurons and astrocytes for the life of the culture, up to 8 weeks (see Fig. 1C). This was generally true whether the cultures were transduced at day 1-2 postplating or at 4 weeks post-plating.

\section{Neuroprotection and neurotoxicity assays in 96-well plates}

Primary hippocampal cultures in a 96-well plate were transduced with AAV1-CMV-GFP to obtain a transduction percentage of $89 \%$ (118/133; see Fig. 3A inset). Cultures were transduced on DIV7 and exposed to increasing concentrations of L-glutamate on DIV14 for $24 \mathrm{hr}$. Transduced cells were imaged digitally under a fluorescent microscope and counted. A doseresponse relationship for glutamate toxicity was observed (Fig. 3A). Pretreatment with the neurotrophin NT-4/5 resulted in dose-dependent protection (Fig. 3B), as previously described [6]. These results demonstrate that AAV transduction can be readily adapted for highthroughput assays.

\section{Discussion}

Current methods for gene transfer into cultured neurons are of limited utility due to low efficiency, high toxicity, short duration of gene expression, or the restriction that gene delivery must be performed in immature cells on the day of plating. Transgenic animals can be a source of genetically modified cultures, but generation of these animals is expensive, labor-intensive, and mostly restricted to mice. Our results demonstrate that the use of AAV vectors can overcome all of these problems. This technique should prove useful for the generation of essentially transgenic neuronal cultures for a variety of neurobiological applications, particularly those requiring biochemical analysis or measurements over the course of weeks. Among many potential applications, AAV transduction of neurons in vitro can be used for neurotoxicity and neuroprotection assays.

Despite their limited capacity (approx. $4.5 \mathrm{~kb}$ ), recombinant AAV viral vectors are capable of delivering a wide variety of useful cassettes for overexpression or downregulation of genes. These include: many common reporter genes; cDNAs linked to constitutively active, cell typespecific or drug-regulated promoters; constitutively expressed shRNA sequences and ribozymes; IRES-GFP or two promoter-cDNA combinations for expression of two genes from 
the same vector; and components of the cre-lox system. In addition, given the high efficiency we observed here, multiple genes may be delivered by using multiple vectors on the same culture with a reasonable expectation of double transduction.

Despite significant differences in capsid sequence, most AAV serotypes tested to date preferentially transduce hippocampal neurons in vivo. Even so, there are notable differences in efficiency and in the subtypes of neurons transduced. In the rat hippocampus, AAV2 primarily transduces hilar interneurons whereas AAV1 and AAV5 preferentially transduce pyramidal neurons in areas CA1-CA3 [3]. AAV8 was demonstrated to transduce pyramidal and dentate granule neurons as well as parvalbumin-containing GABAergic neurons in vivo [17]. Direct comparisons of these serotypes have shown AAV 1 and 8 to be considerably more efficient in the adult rat hippocampus than AAV2 or 5, by immunostaining, Western blot and biophotonic imaging [17]. A comparison of AAV7, 8, 9 and Rh10 in adult mouse hippocampus showed that AAV9 is the most efficient, transducing both CA pyramidal neurons and dentate granule neurons [5]. AAV8 is more efficient for transduction of neonatal mouse brain than AAV1 or AAV2 when injected into the cerebral ventricles [2]. In addition to transducing cell bodies at the site of injection, a few serotypes (e.g. AAV1, 5 and 9) are taken up at axonal terminals and undergo retrograde transport, thereby mediating gene expression in remote cell bodies having axons that terminate in the injected structure $[3,5]$.

To date, more than 100 naturally-occuring isolates of AAV have been identified, allowing for a potentially large variety of target cell types (reviewed in [11]). However, few of the corresponding cellular receptors have been identified. AAV2 binds to heparan sulfate proteoglycans [29] and an integrin-binding domain has also been identified. The hepatocyte growth factor receptor, c-met, $\alpha \mathrm{v} \beta 5$ integrin and FGFR1 have been described as AAV2 coreceptors $[1,15,26,28]$. Sialic acid on cell-surface glycoproteins is important for binding of AAV1, 5 and 6 [31,34], and PDGFR has been identified as an AAV5 receptor [9]. We speculate that differential receptor expression in vitro and in vivo is the most likely explanation for the variation in efficiency and specificity of a given serotype when comparing transduction of the hippocampus in vivo and transduction of dissociated hippocampal neurons in vitro. The AAV serotypes which will be most effective for hippocampal slice cultures should also be determined empirically for this reason.

The promoter also influences the cell-type specificity of gene expression. Kugler and colleagues have shown that gene expression from AAV2 or AAV5 can be restricted to neurons in vitro by incorporating the $\mathrm{hSYN}$ or CBA promoter or restricted mainly to astrocytes by using the mCMV promoter $[18,20,27]$. The hSYN promoter also confers neuronal specificity in vivo [20,27]. Klein, Meyer and colleagues have demonstrated transduction of cultures of astrocytes, microglia and cortical neurons using AAV2 or AAV8 with the CBA promoter $[12,17,18]$. In a quantitative comparison of 10 promoters in AAV2 vectors, in cultures of rat hippocampal, cortical, striatal and nigral cells, the $1.8 \mathrm{~kb}$ NSE promoter and the $2.5 \mathrm{~kb}$ EF1 $\alpha$ promoter mediated the strongest expression; unfortunately, these were two of the largest promoters tested [35]. Even so, the NSE promoter has been used in AAV vectors for efficient and long-term expression of GFP in neurons in vivo [19]. A hybrid hCMV/PDGF $\beta$ promoter and the PDGF $\beta$ promoter alone each showed robust and prolonged neuronal gene expression in vivo $[24,32]$. The hCMV promoter, used here, mediated expression in astrocytes and neurons for weeks in vitro, although it is known to be subject to transcriptional silencing over the course of months in vivo, probably due to methylation $[14,19,22,25]$. Finally, although normal AAVmediated gene expression is detectable within a few days, the process may be accelerated through the use of a self-complementary AAV vector, which presents a more robust and earlier onset of expression in brain in vivo but has only about half the genome packaging capability [21]. 
Our results, together with those cited above, support three general conclusions: (1) that the true tropism of a vector may be masked if the promoter restricts gene expression to a subset of cells; (2) that the tropism of a serotype/promoter combination in vitro is not necessarily predictive of its tropism in vivo; and (3) that the time course of expression is promoter-dependent and can differ between neurons and astrocytes.

We conclude that AAV vectors represent an valuable tool for efficient genetic manipulation of primary neuronal cultures.

\section{Experimental Procedures}

\section{Neuronal Cultures}

The standard culture technique was described previously [8]. Briefly, cortices or hippocampi from E19 Sprague-Dawley rat embryos were trypsinized in Dulbecco's minimum essential medium (DMEM; Whittaker Bioproducts) containing $0.027 \%$ trypsin at $4^{\circ} \mathrm{C}$ for $20 \mathrm{~min}$. They were triturated in media consisting of DMEM supplemented with $10 \%$ bovine calf serum (Hyclone), 10\% Ham's F12 with glutamine (Whittaker Bioproducts), and $50 \mathrm{U} / \mathrm{mL}$ penicillinstreptomycin (Sigma). Dissociated cells were plated on poly-L-lysine coated glass coverslips in $35-\mathrm{mm}$ petri dishes and cultured at $37^{\circ} \mathrm{C}$ in a humidified $5 \% \mathrm{CO} 2$ incubator. Dissociated cells were plated at a density of 100,000 cells/mL in serum-free Neurobasal medium (Gibco) supplemented by B27 (Gibco). Mitotic inhibitors and antibiotics were not used. All procedures involving animals were approved by the University of Pennsylvania Institutional Animal Care and Use Committee and conform to federal guidelines (Guide for the Care and Use of Laboratory Animals, National Research Council, 1996).

\section{AAV vectors}

Recombinant AAV viral vectors were generated by triple transfection of 293 cells and purified by cesium chloride gradient sedimentation as described previously [10]. The plasmid encoding the genome (pZac2.1EGFPWP) was identical for each of the serotypes generated, encoding the AAV2 inverted terminal repeats (ITRs), a human cytomegalovirus (hCMV) immediate early promoter, the enhanced green fluorescent protein (EGFP) coding sequence, a woodchuck hepatitis virus post-transcriptional regulatory element (WPRE) and a bovine growth hormone poly A sequence. This plasmid was cotransfected with plasmids encoding serotype-specific (serotypes 1, 2, 5, 6, 7, 8, and 9) or specifically modified rep and cap sequences. Vector titers were determined by quantitative PCR and typically ranged from $10^{12}$ to $10^{13}$ genome copies $(\mathrm{GC}) / \mathrm{ml}$. In the text, the resulting recombinant vectors are denoted by their serotype number, i.e. AAV1. Multiple independent batches of each CMV-GFP vector were tested: AAV1 $(n=2)$; $\operatorname{AAV} 2(n=5) ; \operatorname{AAV} 5(n=3), \operatorname{AAV} 6(n=2) ; \operatorname{AAV} 7(n=2) ; \operatorname{AAV} 8(n=3) ; \operatorname{AAV} 9(n=2)$. Where noted, the bacterial beta-galactosidase (LacZ) coding sequence replaced GFP and doses were matched to the corresponding GFP vectors: $2.5 \times 10^{11} \mathrm{GC}$ for AAV5 and $6.7 \times 10^{10} \mathrm{GC}$ for AAV6.

Modifications to the AAV2 and AAV6 capsids are described elsewhere ([30] and LV and JWM, manuscript in preparation). Briefly, AAV2 and AAV6 capsids normally bind heparin [13]. AAV2 contains an RXXR consensus sequence (RGNR) on VP3 that mediates heparin binding $[16,23]$. AAVhu29R is a variant of AAV2 capsid with SGNT at the RXXR sequence that blocks heparin binding, in addition to five other single amino acid changes outside the RXXR region [30]. In AAV6, which lacks an RXXR sequence, K531 is a critical residue for heparin binding [33]. AAV6.1 contains a K531E mutation in the AAV6 capsid that ablates heparin binding, and AAV6.2 has a F129L change that does not affect heparin binding (see [33]). AAV6.1.2 contains both the K513E and F129L changes and does not bind heparin. 
Vectors were concentrated by banding in cesium chloride, then collected in PBS containing $5 \%$ glycerol, stored in aliquots at $-80^{\circ} \mathrm{C}$ and thawed on ice before use. Volumes not exceeding $50 \mu \mathrm{l}$ (corresponding to doses listed in Table 1) were added to 35 -mm plates containing 150,000 cells in $1.5 \mathrm{ml}$ medium. Control cultures received vehicle only (PBS $+5 \%$ glycerol) or no treatment.

\section{Immunofluorescence analysis and cell counts}

Cells were fixed with $10 \%$ neutral buffered formalin (10 min RT), permeabilized with Trisbuffered saline (TBS) containing 0.3\% Triton (TBST), blocked in 5\% goat or donkey serum in TBS, exposed to the primary antibody overnight at $4{ }^{\circ} \mathrm{C}$, washed and incubated with the corresponding secondary antibody, washed, incubated with the fluorescent streptavidin ( $1 \mathrm{hr}$ RT each), then washed again and inverted onto glass slides with Vectashield mounting media containing DAPI (Vector Laboratories) to identify nuclei. Neurons were identified by immunostaining with rabbit polyclonal anti-MAP2 (1:500, gift of Dr. Virginia Lee, University of Pennsylvania), and detected with biotin-SP-conjugated goat anti-rabbit IgG and Alexa594conjugated streptavidin (Molecular Probes, 1:500 each). Astrocytes were identified on separate coverslips with monoclonal anti-GFAP antibody (Sigma, 1:500), biotin-SP-conjugated donkey anti-mouse IgG and Alexa594-conjugated streptavidin (Molecular Probes, 1:500 each). The specificity of staining was confirmed by omitting the primary antibody in each case.

Immunofluorescence was colocalized with intrinsic GFP fluorescence. Digital images in each channel were acquired with a Nikon $80 \mathrm{i}$ microscope, a CoolSnap cf2 monochrome camera and ImagePro software, and pseudocolored appropriately (green for GFP; red for MAP2 or GFAP; blue for DAPI). Manual cell counts were performed by two observers on digital images of 4 10 randomly selected, nonoverlapping single-channel $10 \times$ fields. To calculate the overall percent transduction, the number of GFP-expressing cells was expressed as a percentage of nuclei stained with DAPI (GFP+/DAPI+). To calculate the percentage of neurons in the transduced population, the formula was MAP2+GFP+/GFP+. Statistical comparisons were made between AAV1 and either AAV2, 7, 8 or 9 using Student's $t$ test.

Toxicity was assessed by inspection of the MAP2 staining, using the following scale: $0=$ healthy cultures with uniform MAP2 staining in the processes; $1=$ beaded MAP2 staining in the processes but little or no cell loss; 2 = substantial cell loss and/or fragmented MAP2 staining in the processes and cell body; 3 = majority of cells dead. Transduction was quantified in a culture only if the toxicity was rated as 0 or 1 . MAP2 has been used in similar assays by other groups [4]. Severe toxicity (grade 2 or 3 ) was present in all of the cultures treated for 2 weeks with AAV5-CMV-GFP ( $\mathrm{n}=2$ of 2 cultures at $\left.2.5 \times 10^{11} \mathrm{GC}\right)$, AAV6-CMV-GFP $(\mathrm{n}=9 / 9$ at $6.7 \times 10^{10} \mathrm{GC}$ and above), AAV6.2 (3/3 at 5.0 $\left.\times 10^{10} \mathrm{GC}\right)$, AAV5-CMV-LacZ $(\mathrm{n}=3 / 3$ at $\left.2.5 \times 10^{11} \mathrm{GC}\right)$, AAV6-CMV-LacZ ( $\mathrm{n}=3 / 3$ at $6.7 \times 10^{10} \mathrm{GC}$ ). Occasional toxicity was observed in cultures treated with the other serotypes at the highest dose of $2.0-2.5 \times 10^{11}:$ AAV1 $(n=1$ of 5 cultures); AAV2 (1/6); AAV7(1/6); AAV8 (1/6) and AAV9 (n=0/4).

\section{Neurotoxicity and neuroprotection assays}

Dissociated primary hippocampal neurons $(100,000$ cells/ml $)$ were plated in poly-L-lysinecoated 96-well plates. One week after plating, each well was transduced with AAV1 CMVGFP, MOI $1.47 \times 10^{5}$. Two weeks after plating, cells were exposed to L-glutamate $(0-400$ $\mu \mathrm{M}) .24$ hours following treatment with L-glutamate, cells were fixed with $10 \%$ neutral buffered formalin (10 min RT) and mounted with immumount. For neuroprotection experiments, cells were pre-treated with rhNT-4/5 (0-1000 ng/ml; R\&D Systems) 24 hours prior to treatment with 3.7 or $11.1 \mu \mathrm{M}$ L-glutamate. Digital imaging was performed as described above. Manual cell counts were performed on each image, three non-overlapping $10 \times$ fields per well and three wells per condition. Cell survival was calculated as percentage of the untreated wells. 


\section{Acknowledgements}

This research was supported by grants from the National Institutes of Health RO1 NS040978 (DJW), P30 DK 47757-09 and P01 HL 59407-03 (JMW) and RO1 NS24260 (MAD), by a pilot grant from the University of Pennsylvania Institute for Medicine and Engineering (DJW) and by startup funds from the University of Pennsylvania Department of Neurosurgery (DJW). We thank the Vector and Quality Control Cores of the University of Pennsylvania for producing the vectors, Dr. Virginia Lee for the MAP2 antibody, Drs. Dennis Kolson and Lauren O'Donnell for valuable advice about preparing 96-well plates for primary cultures, Dr. Robert Neumar for assistance with photography, and Dr. M. Sean Grady for mentorship and research support. JWM holds equity in Targeted Genetics Corp.

\section{References}

1. Asokan A, Hamra JB, Govindasamy L, Agbandje-McKenna M, Samulski RJ. Adeno-associated virus type 2 contains an integrin alpha5beta1 binding domain essential for viral cell entry. J Virol 2006;80:8961-8969. [PubMed: 16940508]

2. Broekman ML, Comer LA, Hyman BT, Sena-Esteves M. Adeno-associated virus vectors serotyped with AAV8 capsid are more efficient than AAV-1 or -2 serotypes for widespread gene delivery to the neonatal mouse brain. Neurosci 2006;138:501-510.

3. Burger C, Gorbatyuk OS, Velardo MJ, Peden CS, Williams P, Zolotukhin S, Reier PJ, Mandel RJ, Muzyczka N. Recombinant AAV viral vectors pseudotyped with viral capsids from serotypes 1, 2, and 5 display differential efficiency and cell tropism after delivery to different regions of the central nervous system. Mol Ther 2004;10:302-317. [PubMed: 15294177]

4. Carrier RL, Ma TC, Obrietan K, Hoyt KR. A sensitive and selective assay of neuronal degeneration in cell culture. J Neurosci Meth 2006;154:239-244.

5. Cearley C, Wolfe JH. Transduction characteristics of adeno-associated virus vectors expressing cap serotypes 7, 8, 9 and Rh10 in the mouse brain. Mol Ther 2006;13:528-537. [PubMed: 16413228]

6. Cheng B, Goodman Y, Begley JG, Mattson MP. Neurotrophin-4/5 protects hippocampal and cortical neurons against energy deprivation- and excitatory amino acid-induced injury. Brain Res 1994;650:331-335. [PubMed: 7953701]

7. Craig, AM. Transfecting cultured neurons. In: Banker, G.; Goslin, K., editors. Culturing nerve cells. MIT Press; Cambridge, MA: 1998. p. 79-111.

8. Cummings DD, Wilcox KS, Dichter MA. Calcium-dependent paired-pulse facilitation of miniature EPSC frequency accompanies depression of EPSCs at hippocampal synapses in culture. J Neurosci 1996;16:5312-5323. [PubMed: 8757244]

9. Di Pasquale G, Davidson BL, Stein CS, Martins I, Scudiero D, Monks A, Chiorini J. Identification of PDGFR as a receptor for AAV-5 transduction. Nat Med 2003;9:1306-1312. [PubMed: 14502277]

10. Fisher KJ, Jooss K, Alston J, Yang Y, Haecker SE, High K, Pathak R, Raper SE, Wilson JM. Recombinant adeno-associated virus for muscle directed gene therapy. Nat Med 1997;3:306-312. [PubMed: 9055858]

11. Gao G, Vandenberghe LH, Wilson JM. New recombinant serotypes of AAV vectors. Curr Gene Ther 2005;5:285-297. [PubMed: 15975006]

12. Gong Y, Chen S, Sonntag CF, Sumners C, Klein RL, King MA, Hughes JA, Meyer EM. Recombinant adeno-associated virus serotype 2 effectively transduces primary rat brain astrocytes and microglia. Brain Res Protocols 2004;14:18-24.

13. Halbert CL, Allen JM, Miller AD. Adeno-associated virus type 6 (AAV6) vectors mediate efficient transduction of airway epithelial cells in mouse lungs compared to that of AAV2 vectors. J Virol 2001;75:6615-6624. [PubMed: 11413329]

14. Kaplitt MG, Leone P, Samulski RJ, Xiao X, Pfaff DW, O’Malley KL, During MJ. Long-term gene expression and phenotypic correction using adeno-associated virus vectors in the mammalian brain. Nat Gen 1994;8:148-154.

15. Kashiwakura Y, Tamayose K, Iwabuchi K, Hirai Y, Shimada T, Matsumoto K, Nakamura T, Watanabe M, Oshimi K, Daida H. Hepatocyte growth factor receptor is a coreceptor for adenoassociated virus type 2 infection. J Virol 2005;79:609-614. [PubMed: 15596854]

16. Kern A, Schmidt K, Leder C, Muller OJ, Wobus CE, Bettinger K, Von der Lieth CW, King JA, Kleinschmidt JA. Identification of a heparin-binding motif on adeno-associated virus type 2 capsids. J Virol 2003;77:11072-11081. [PubMed: 14512555] 
17. Klein RL, Dayton RD, Leidenheimer NJ, Jansen K, Golde TE, Zweig RM. Efficient neuronal gene transfer with AAV8 leads to neuroroxic levels of tau or green fluorescent proteins. Mol Ther 2006;13:517-526. [PubMed: 16325474]

18. Klein RL, Hamby ME, Gong Y, Hirko AC, Wang S, Hughes JA, King MA, Meyer EM. Dose and promoter effects of adeno-associated viral vector for green fluorescent protein expression in the rat brain. Exp Neurol 2002;176:66-74. [PubMed: 12093083]

19. Klein RL, Meyer EM, Peel AL, Zolotukhin S, Meyers C, Muzyczka N, King MA. Neuron-specific transduction in the rat septohippocampal or nigrostriatal pathway by recombinant adeno-associated virus vectors. Exp Neurol 1998;150:183-194. [PubMed: 9527887]

20. Kugler S, Lingor P, Scholl U, Zolotukhin S, Bahr M. Differential transgene expression in brain cells in vivo and in vitro from AAV-2 vectors with small transcriptional control units. Virol 2003;311:8995.

21. McCarty DM, Fu H, Monahan PE, Toulson CE, Naik P, Samulski RJ. Adeno-associated virus terminal repeat (TR) mutant generates self-complementary vectors to overcome the rate-limiting step to transduction in vivo. Gene Ther 2003;10:2112-2118. [PubMed: 14625565]

22. McCown TJ, Xiao X, Li J, Breese GR, Samulski RJ. Differential and persistent expression patterns of CNS gene transfer by an adeno-associated virus (AAV) vector. Brain Res 1996;713:99-107. [PubMed: 8724980]

23. Opie SR, Warrington KH Jr, Agbandje-McKenna M, Zolotukhin S, Muzyczka N. Identification of amino acid residues in the capsid proteins of adeno-associated virus type 2 that contribute to heparan sulfate proteoglycan binding. J Virol 2003;77:6995-7006. [PubMed: 12768018]

24. Paterna JC, Moccetti T, Mura A, Feldon J, Bueler H. Influence of promoter and WHV posttranscriptional regulatory element on AAV-mediated transgene expression in the rat brain. Gene Ther 2000;7:1304-1311. [PubMed: 10918501]

25. Prosch S, Stein J, Staak K, Liebenthal C, Volk HD, Kruger DH. Inactivation of the very strong HCMV immediate early promoter by DNA CpG methylation in vitro. Biol Chem Hoppe-Seyler 1996;377:195-201. [PubMed: 8722321]

26. Qing K, Mah C, Hansen J, Zhou S, Dwarki V, Srivastava A. Human fibroblast growth factor receptor 1 is a co-receptor for infection by adeno-associated virus 2. Nat Med 1999;5:71-77. [PubMed: 9883842]

27. Shevtsova Z, Malik JM, Michel U, Bahr M, Kugler S. Promoters and serotypes: targeting of adenoassociated virus vectors for gene transfer in the rat central nervous system in vitro and in vivo. Exp Physiol 2005;90:53-59. [PubMed: 15542619]

28. Summerford C, Bartlett JS, Samulski RJ. AlphaVbeta5 integrin: a co-receptor for adeno-associated virus type 2 infection. Nat Med 1999;5:78-82. [PubMed: 9883843]

29. Summerford C, Samulski RJ. Membrane-associated heparan sulfate proteoglycan is a receptor for adeno-associated virus type 2 virions. J Virol 1998;72:1438-1445. [PubMed: 9445046]

30. Vandenberghe LH, Wang L, Somanathan S, Zhi Y, Figueredo J, Calcedo R, Sanmiguel J, Desai RA, Chen CS, Johnston J, Grant RL, Gao G, Wilson JM. Heparin binding directs activation of T cells against adeno-associated virus serotype 2 capsid. Nat Med 2006;12:967-971. [PubMed: 16845388]

31. Walters RW, Yi SM, Keshavjee S, Brown KE, Welsh MJ, Chiorini JA, Zabner J. Binding of adenoassociated virus type 5 to 2,3-linked sialic acid is required for gene transfer. $\mathrm{J}$ Biol Chem 2001;276:20610-20616. [PubMed: 11262413]

32. Wang CY, Guo HY, Lim TM, Ng YK, Neo HP, Hwang PY, Yee WC, Wang S. Improved neuronal transgene expression from an AAV-2 vector with a hybrid CMV enhancer/PDGF-beta promoter. J Gene Med 2005;7:945-955. [PubMed: 15756650]

33. Wu Z, Asokan A, Grieger J, Govindasamy L, Agbandje-McKenna M, Samulski RJ. Single amino acid changes can influence titer, heparin binding, and tissue tropism in different adeno-associated virus serotypes. J Virol 2006b;80:11393-11397. [PubMed: 16943302]

34. Wu Z, Miller E, Agbandje-McKenna M, Samulski RJ. Alpha2,3 and alpha2,6 N-linked sialic acids facilitate efficient binding and transduction by adeno-associated virus types 1 and 6. J Virol 2006a; 80:9093-9103. [PubMed: 16940521]

35. Xu R, Janson CG, Mastakov M, Lawlor P, Young D, Mouravlev A, Fitzsimons H, Choi KL, Ma H, Dragunow M, Leone P, Chen Q, Dicker B, During MJ. Quantitative comparison of expression with 
adeno-associated virus (AAV-2) brain-specific gene cassettes. Gene Ther 2001;8:1323-1332. [PubMed: 11571569]

\section{Abbreviations}

AAV

adeno-associated viral vector

CBA

chicken beta-actin

$\mathrm{CsCl}$

cesium chloride

DAPI

4',6-diamidino-2-phenylindole

DIV

days in vitro

DMEM

Dulbecco's modified Eagle's medium

EF1a

elongation factor 1 alpha

FGFR1

fibroblast growth factor receptor 1

GC

genome copies

GFAP

glial fibrillary acidic protein

GFP

green fluorescent protein

hCMV

human cytomegalovirus

IRES

internal ribosomal entry site

NT-4/5

neurotrophin-4/5

NSE

neuron-specific enolase

PBS

phosphate buffered saline

SiRNA

small interfering RNA

SV40

Simian virus 40

Brain Res. Author manuscript; available in PMC 2009 January 23. 


\section{SYN}

synuclein

TBS

Tris-buffered saline

TBS-T

Tris buffered saline with Triton

MAP2

microtubule-associated protein 2

WPRE

woodchuck hepatitis virus posttranslational regulatory element 

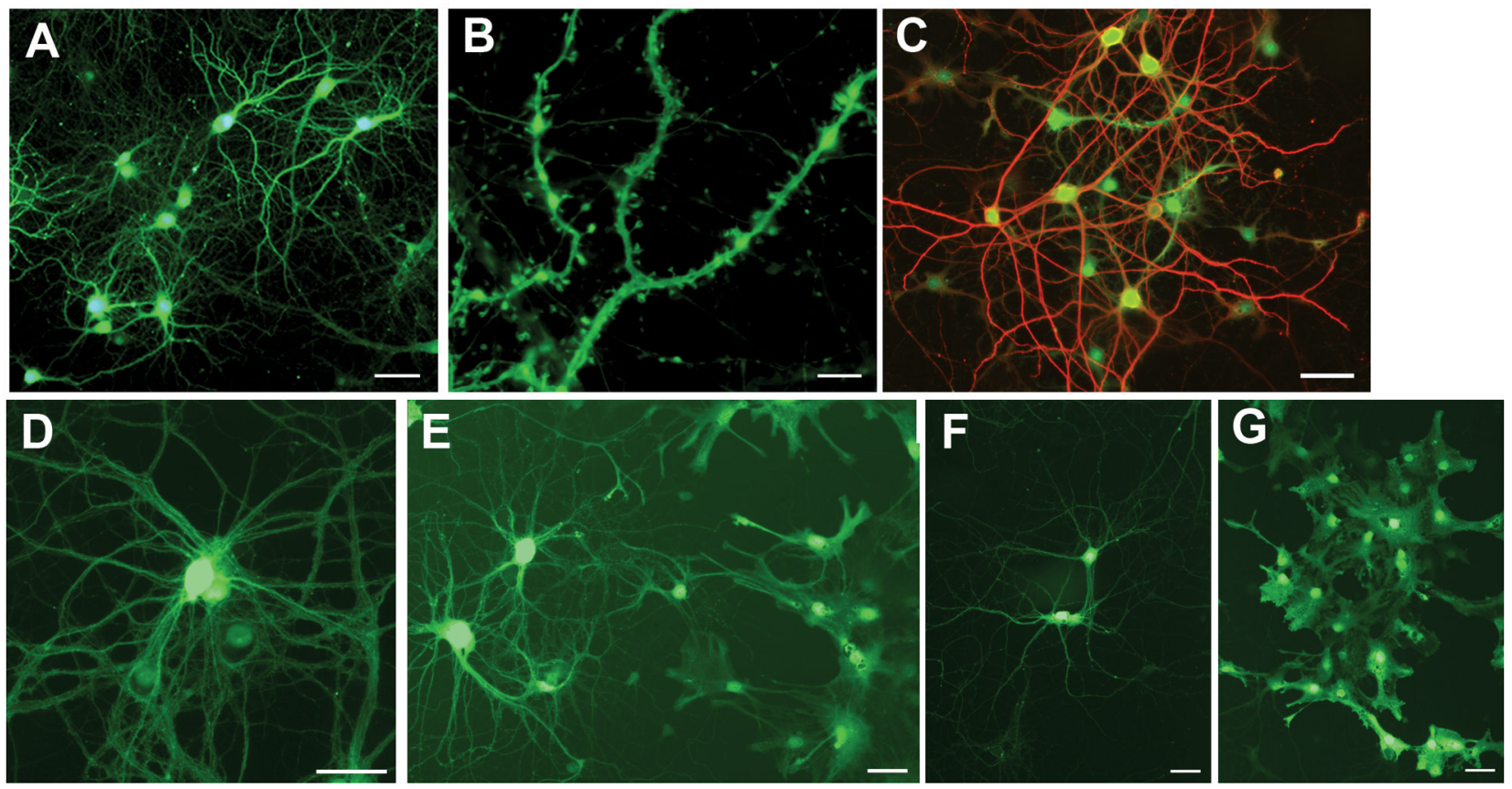

Figure 1.

Transduction of cultured neurons and astrocytes by AAV serotypes. (A-B) Hippocampal cultures transduced by AAV2 at 4 weeks in vitro and harvested at 5 weeks in vitro. Green is intrinsic GFP fluorescence. (C) Hippocampal cultures transduced by AAV2 at 4 weeks in vitro and harvested at 8 weeks in vitro. Green is intrinsic GFP fluorescence; red is MAP2 immunostaining for neurons. (D-G) Cortical cultures transduced at 4 weeks in vitro and harvested at 5 weeks in vitro. Green is intrinsic GFP fluorescence. (D) AAV1 transduction of neurons. (E) AAV7 transduction of neurons (left) and astrocytes (right). (F) AAV9 transduction of neurons. (G) AAV9 transduction of astrocytes. Bar in A, C-G, 50 microns. Bar in B, 5 microns. 


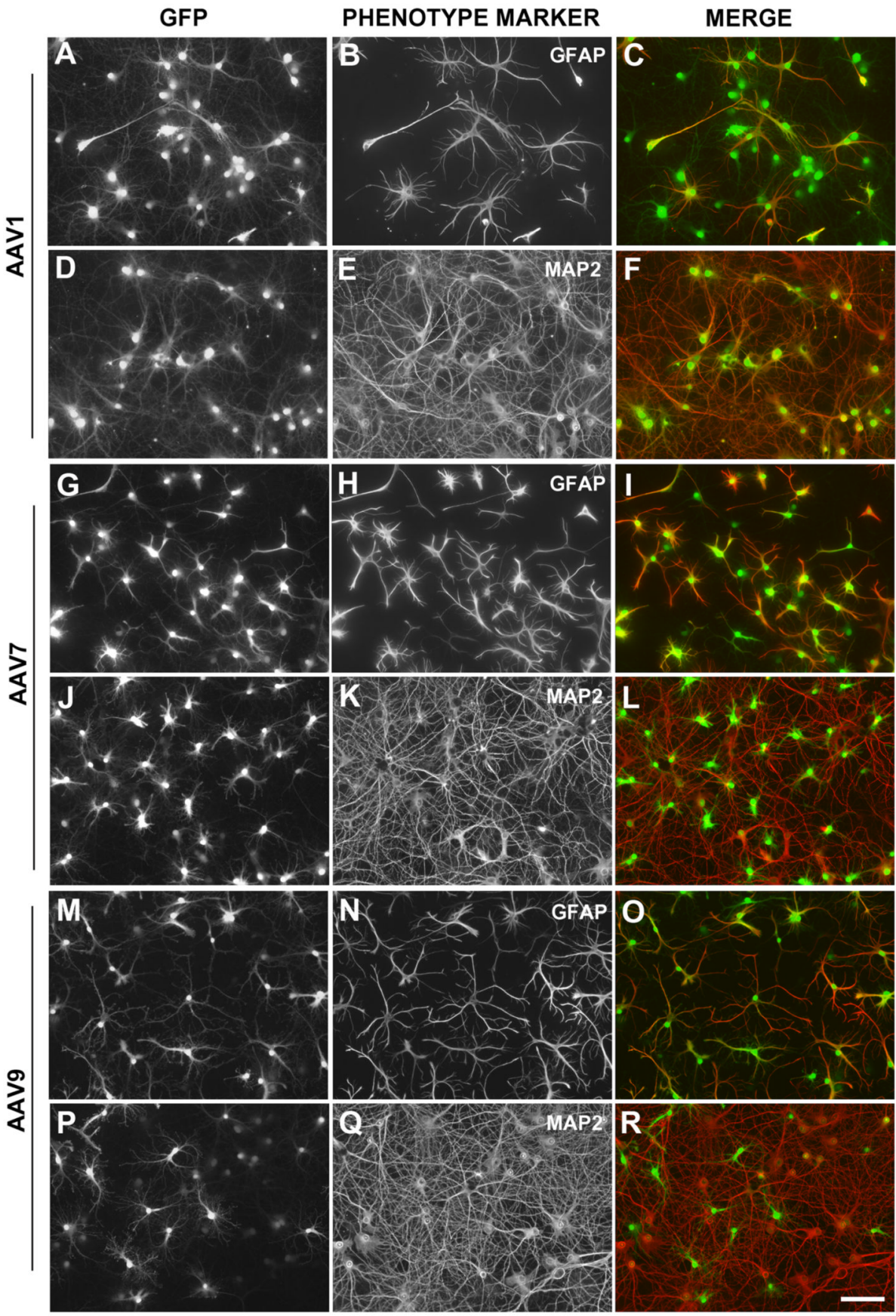

Figure 2.

Expression of GFP in neurons and astrocytes of hippocampal cultures transduced by AAV1 (A-F), AAV7 (G-L), or AAV9 (M-R). GFP expression is shown in the first column (A, D, G, $\mathrm{J}, \mathrm{M} \mathrm{R})$ and GFAP immunostaining $(\mathrm{B}, \mathrm{H}, \mathrm{N})$ or MAP2 immunostaining $(\mathrm{E}, \mathrm{K}, \mathrm{Q})$ in the second column. The third column contains a merged pseudocolored representation of the first two panels in each row. Bar, 100 microns. 

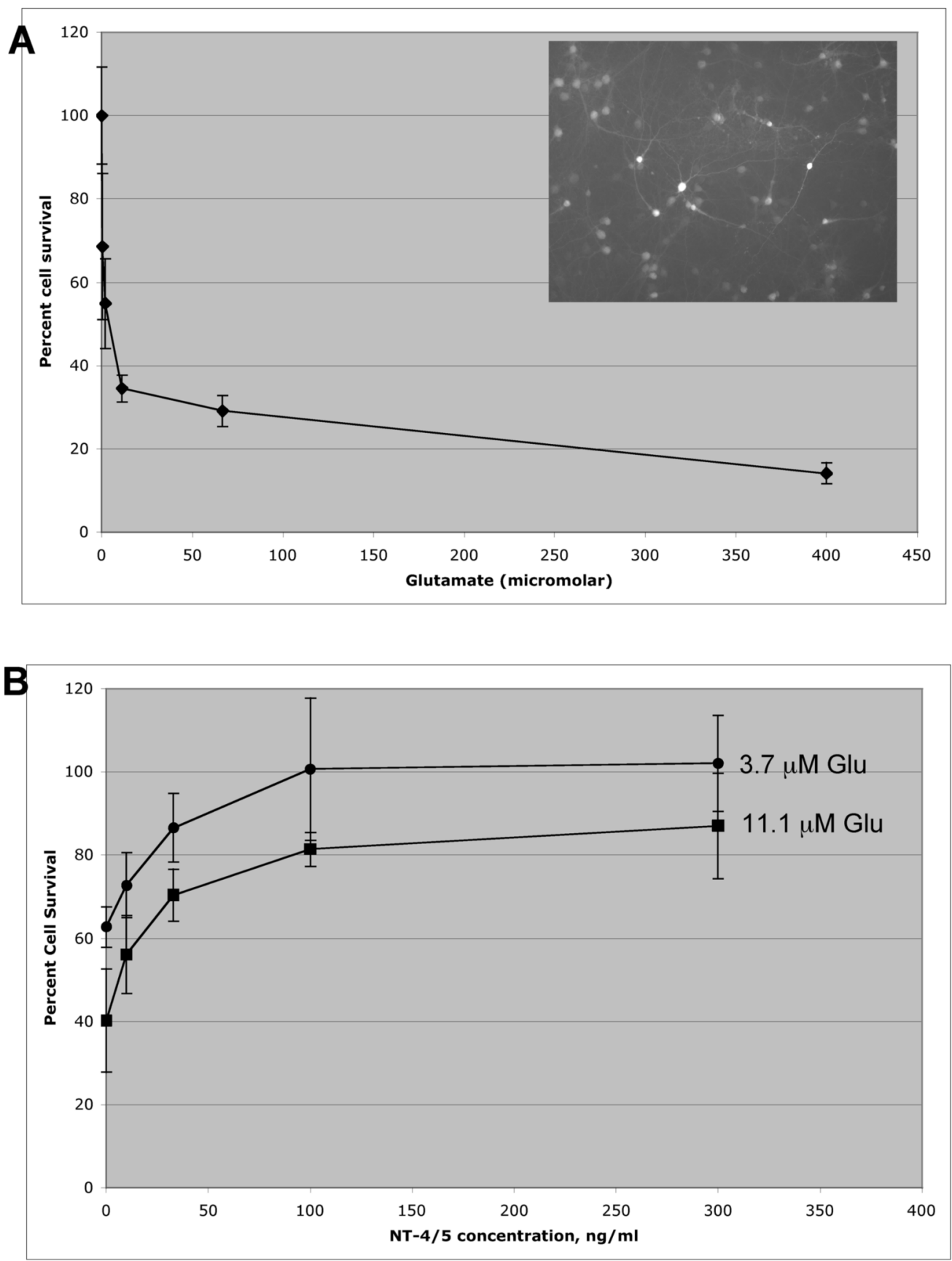

Figure 3.

Glutamate toxicity and NT-4/5 protection in AAV1-transduced primary hippocampal cultures in a 96-well plate. Cultures were transduced at DIV7 with AAV1-CMV-GFP. (A) Increasing concentrations of L-glutamate $(0,0.3,1.9,11.1,66.6$ or 400 microM) were added to wells $(n=3$ per dose) at DIV14 for $24 \mathrm{hr}$. Values are normalized to the untreated well (100\%). Inset, monochrome fluorescent photomicrograph of transduced (GFP expressing) cells in a 96-well plate. (B) Cultures were treated with increasing concentrations of NT-4/5 $(0,10,33,100$ or $300 \mathrm{ng} / \mathrm{ml}$ ) for $24 \mathrm{hr}$ before exposure to $3.7 \mathrm{mM}$ (filled circles) or $11.1 \mathrm{mM}$ (filled squares) of L-glutamate for $24 \mathrm{hr}$. 


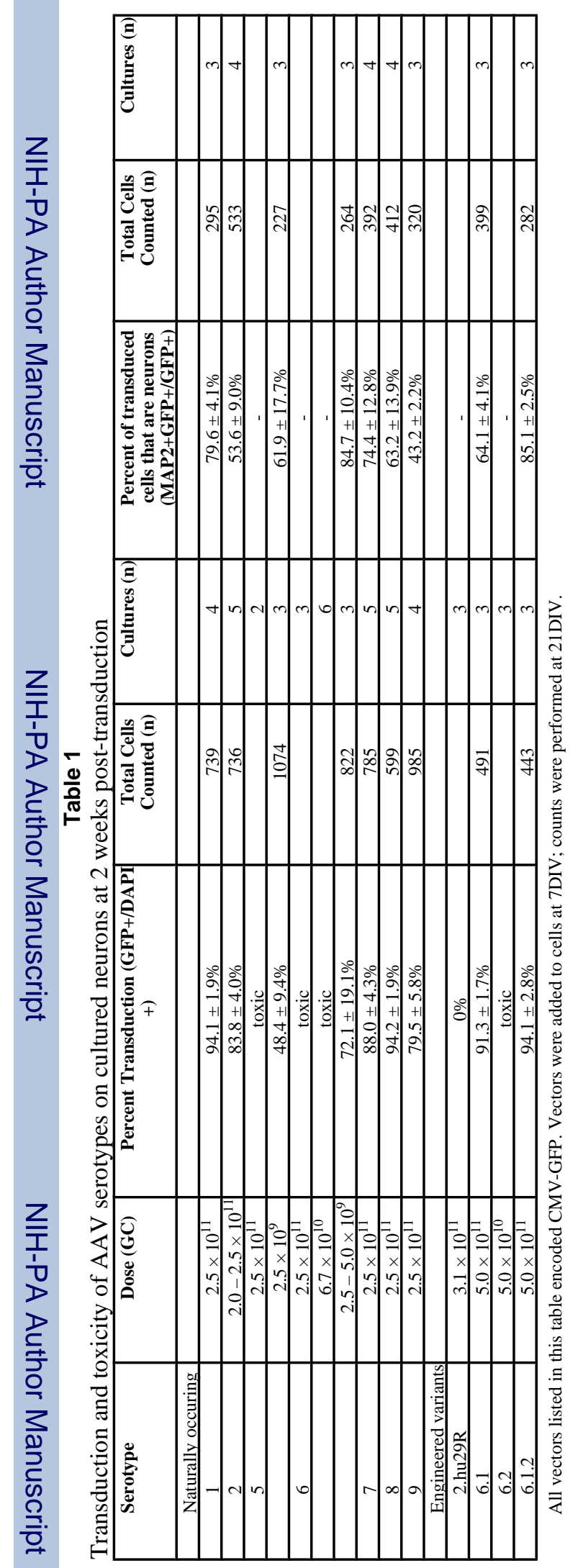

Brain Res. Author manuscript; available in PMC 2009 January 23. 